CORRECTION

\title{
Correction: Association of low-frequency and rare coding variants with information processing speed
}

Jan Bressler (D), Gail Davies (D), Albert V. Smith, Yasaman Saba, Joshua C. Bis, Xueqiu Jian, Caroline Hayward (D), Lisa Yanek (D), Jennifer A. Smith (D), Saira S. Mirza, Ruiqi Wang, Hieab H. H. Adams (D), Diane Becker, Eric Boerwinkle, Archie Campbell, Simon R. Cox (D), Gudny Eiriksdottir, Chloe Fawns-Ritchie, Rebecca F. Gottesman (D), Megan L. Grove (D), Xiuqing Guo, Edith Hofer, Sharon L. R. Kardia, Maria J. Knol (D), Marisa Koini, Oscar L. Lopez (D), Riccardo E. Marioni, Paul Nyquist, Alison Pattie, Ozren Polasek, David J. Porteous, Igor Rudan, Claudia L. Satizabal (D), Helena Schmidt, Reinhold Schmidt, Stephen Sidney, Jeannette Simino, Blair H. Smith (D), Stephen T. Turner, Sven J. van der Lee (D), Erin B. Ware, Rachel A. Whitmer, Kristine Yaffe, Qiong Yang, Wei Zhao (iD, Vilmundur Gudnason, Lenore J. Launer (D), Annette L. Fitzpatrick, Bruce M. Psaty, Myriam Fornage (D), M. Arfan Ikram (D), Cornelia M. van Duijn, Sudha Seshadri, Thomas H. Mosley and lan J. Deary (iD

(c) The Author(s) 2022

Translational Psychiatry (2022)12:88; https://doi.org/10.1038/s41398-022-01852-x

Correction to: Translational Psychiatry https://doi.org/10.1038/ s41398-021-01736-6, published online 4 December 2021

The original version of this article unfortunately contained mistakes in the author affiliations. The original article has been corrected.

The correct affiliations are as follows:

Eric Boerwinkle ${ }^{1,19}$, Archie Campbell $20,21,22$, Rebecca F. Gottesman $^{23,24}$, Megan L. Grove ${ }^{1}$, Xiuqing Guo ${ }^{25}$, Edith Hofer ${ }^{26,27}$, Maria J. $\mathrm{Knol}^{13}$, Marisa Koini ${ }^{26}$, Oscar L. Lopez ${ }^{28,29}$, Riccardo E. Marioni ${ }^{20}$, Paul Nyquist ${ }^{23}$, Ozren Polasek ${ }^{30,31}$, David J. Porteous ${ }^{20,32}$, Igor Rudan ${ }^{33}$, Claudia L. Satizibal ${ }^{17,34,35}$, Reinhold Schmidt ${ }^{26}$, Stephen Sidney ${ }^{36}$, Jeannette Simino ${ }^{37}$, Blair H. Smith ${ }^{38}$, Stephen T. Turner ${ }^{39}$, Sven J. van der Lee ${ }^{13}$, Erin B. Ware ${ }^{12}$, Rachel A. Whitmer ${ }^{36}$, Kristine Yaffe $^{40}$, Vilmundur Gudnason ${ }^{4,41}$, Lenore J. Launer ${ }^{42}$, Annette L.
Fitzpatrick $^{43,44}$, Bruce M. Psaty ${ }^{6,44,45}$, M. Arfan Ikram ${ }^{13}$, Cornelia M. van Duijn ${ }^{13,46}$, Sudha Seshadri ${ }^{17,34,35}$, Thomas H. Mosley ${ }^{47}$.

\begin{abstract}
(C) Open Access This article is licensed under a Creative Commons Attribution 4.0 International License, which permits use, sharing, adaptation, distribution and reproduction in any medium or format, as long as you give appropriate credit to the original author(s) and the source, provide a link to the Creative Commons license, and indicate if changes were made. The images or other third party material in this article are included in the article's Creative Commons license, unless indicated otherwise in a credit line to the material. If material is not included in the article's Creative Commons license and your intended use is not permitted by statutory regulation or exceeds the permitted use, you will need to obtain permission directly from the copyright holder. To view a copy of this license, visit http://creativecommons. org/licenses/by/4.0/.
\end{abstract}

(c) The Author(s) 2022 